\title{
Synthetic Pyrethroids: Toxicity and Metabolism
}

\author{
Meet Patel, Piyusha Patil \\ (Department of Dyestuff Technology, Institute of Chemical Technology, India) \\ (Department of Food Engineering and Technology, Institute of Chemical Technology, India)
}

\begin{abstract}
Synthetic Pyrethroids are synthetic analogs of naturally occurring pyrethrins, found in extract from Chrysanthemum flowers. They were launched as a replacement of highly toxic class of pesticides like DDT and Organophosphates in 1980s. Major success of pyrethroids lies in the low toxicity of them towards mammals as well as humans and due to which they have wide range of applications right from agricultural fields, as household pesticides to veterinary applications. Low toxicity of pyrethroids is mainly defined by high rate of metabolism reaction via ester hydrolysis and oxidation at certain position depending on the structure which are mediated by carboxylesterase and CYP isoforms. These metabolites further undergoes either hydrophilic or lipophilic conjugate formation based on the structure of molecule. One of the drawback of pyrethroids is toxicity to aquatic organism and bees which limits their use for crops like paddy, chestnut etc.
\end{abstract}

Keywords: pyrethroids, toxicity, synergists, metabolism, cyhalothrin

\section{Introduction}

Insecticidal activity of natural pyrethrins which was one of the constituent of pyrethrum extract obtained from chrysanthemum flower have been known to mankind since ancient times. In 1920s U.S. military used to formulate pyrethrum extract powder as space spray for use against houseflies and mosquitoes. ${ }^{[9]}$ The problem with pyrethrin was its photo-stability which limits its use in open agriculture field. Breakthrough in 1970s eventually led to the development of synthetic pyrethroids with high photo-stability and high insecticidal activities with high knockdown rate. These developments helped increase the range of application to agriculture fields. Pyrethroids are neurotoxic poison and targets voltage-sensitive sodium channels. In addition to these, pyrethroids mainly of Type II also affects voltage-gated calcium channels, voltage-gated chloride channel and $\mathrm{GABA}_{\mathrm{A}}$ receptors. ${ }^{[6]}$

Synthetic pyrethroids can be divided into first generation and second generation. First generation pyrethroids are mainly ester of chrysanthemic acid derivatives and alcohols with furan ring with terminal side change moiety, these class of pyrethroids are extremely sensitive to light and air oxidation. While second generation pyrethroids with 3-phenoxybenzyl alcohol as one of the moiety is quite photo-stable and thus can be used against agricultural pest. Further second generation pyrethroids can be divided into type I and type II pyrethroids, with latter containing cyano group and thus increasing its insecticidal properties. Type I pyrethroids are generally used as household pesticides, veterinary medicines because of their low photo-stability while type II can be used even as agriculture pesticides.

Table 1.1: Commercially important pyrethroids ${ }^{[5]}$

\begin{tabular}{|c|c|}
\hline Type I & Type II \\
\hline Allenthrin & Cypermethrin \\
Resmethrin & Cyhalothrin \\
Tetramethrin & Fenvalerate \\
Permethrin & Flumethrin \\
& Fluvalinate \\
\hline
\end{tabular}

Toxicity studies have always been given importance while considering pyrethroids. Toxicity to mammals, humans, and environment, aquatic animals is well reported in past years. During initial development of pyrethroids, various studies related to type 1 pyrethroids were undertaken by companies like Sumitomo Chemical Co. etc. Further development in structures of pyrethroids led to in vivo and in vitro studies of various metabolism reaction for newly developed second generation pyrethroids. Also chirality was main attention of study while considering metabolic and toxicology studies of synthetic pyrethroids. Beginning of $21^{\text {st }}$ century led to foundation of molecular biology which helped to study CYP isoforms or Carboxylesterase of animals as well as humans. And thus it was possible to determine which enzymes are responsible for metabolic reactions and clearly detect species difference between humans and laboratory animals. This paper aims to contribute to toxicology data of various pyrethroids, compare these data with other classes of insecticides like DDT and Organophosphorus and recent development in study of metabolic reactions of synthetic pyrethroids. ${ }^{[1]}$ 


\section{Toxicity of Pyrethroids}

Pyrethroids show almost negligible acute toxicity to mammals but are highly toxic to target organism. Studies have shown the main effects of pyrethroids as neurotoxicity at high doses and liver hypertrophy which are reversible if death does not occur. Table 2 clear shows $\mathrm{LD}_{50}$ value of some of the pyrethroids to be on a higher side.

Table 2: $\mathrm{LD}_{50}$ values ${ }^{[4]}$

\begin{tabular}{|c|c|c|}
\hline Insecticides & Oral $\mathrm{LD}_{50}(\mathrm{rats})(\mathrm{mg} / \mathrm{kg})$ & Dermal $\mathrm{LD}_{50}(\mathrm{rats})(\mathrm{mg} / \mathrm{kg})$ \\
\hline Deltamethrin & $531-5000$ & $>2000$ \\
\hline Cypermethrin & $251-4123$ & $>2400(\mathrm{rabbit})$ \\
\hline Bioresmethrin & $7070-8000$ & $>10000(\mathrm{female})$ \\
\hline Allenthrin & $1100(\mathrm{male})$ & $>2300$ \\
\hline
\end{tabular}

\subsection{Comparison with other insecticides}

Pyrethroids were introduced as a replacement of other class of insecticides like DDT and Organophosphorus. The problem with previously used insecticides was their toxicity to non-target organisms especially mammals and humans. These problem was solved by pyrethroids with its low toxicity to humans and mammals. As seen in table 2.1 the selectivity pyrethroids was much more towards target organisms with respect to other insecticides.

Table 2.1: Selectivity Comparison ${ }^{[9]}$

\begin{tabular}{|c|c|c|c|}
\hline Insecticides & \multicolumn{2}{|c|}{$\mathrm{LD}_{50}(\mathrm{mg} / \mathrm{kg})$} & Selectivity \\
& Rat & Insects & \\
\hline Carbamate & $45(15)$ & $2.8(27)$ & 16 \\
\hline Organophosphorus & $67(83)$ & $2.0(50)$ & 33 \\
\hline Organochlorine & $230(21)$ & $2.6(26)$ & 91 \\
\hline Pyrethroids & $2000(27)$ & $0.45(35)$ & 4500 \\
\hline
\end{tabular}

*Number of insecticides tested in parenthesis

For example, the average $\mathrm{LD}_{50}$ value of 15 carbamates for rats is $45 \mathrm{mg} / \mathrm{kg}$, whereas for 27 carbamates for insects it is $2.8 \mathrm{mg} / \mathrm{kg}$. Accordingly, the $\mathrm{LD}_{50}$ value for mammals and insects, an index of selective toxicity, is 16 . The corresponding value of organophosphorus compounds is 33 , and that of organochlorine compounds is 91. In contrast, the value of pyrethroids is 4,500 , indicating much lower toxicity to mammals in spite of their excellent insecticidal activity.

\subsection{Toxicity to aquatic animals}

Aquatic animals are highly sensitive to pyrethroid poisoning from even a small concentrations. Because of high fish toxicity, the use of pyrethroids is prohibited or greatly restricted in and around water systems; for example, in rooms with a water tank containing pet fish, or in and around paddy fields and fishponds. High toxicity to fish can be explained due to low metabolism rate of pyrethroids via ester hydrolysis in the body.

Table 2.2: $\mathrm{LC}_{50}$ value for fish ${ }^{[9]}$

\begin{tabular}{|c|c|c|}
\hline Pyrethroid & \multicolumn{2}{|c|}{$\begin{array}{c}\mathrm{LC}_{50} \text { Value } \\
\text { Daphnid }(\mathrm{ppm} / 3 \mathrm{~h})\end{array}$} \\
\hline Silafluofen & $>100$ & 7.66 \\
\hline Cycloprothrin & 8 & $>10$ \\
\hline Fenvalerate & 0.00075 & 0.3 \\
\hline Cyfluthrin & 0.012 & 0.94 \\
\hline Fluvalinate & 0.00048 & 0.298 \\
\hline
\end{tabular}

As we can see from table 2.2, except recently developed silafluofen, all other pyrethroids are toxic to fish. Apart from aquatic animals, pyrethroids are toxic to some of the non-target organisms like bees, which is seen in table 2.3

Table 2.3: $\mathbf{L C}_{50}$ for bees ${ }^{[9],[2]}$

\begin{tabular}{|c|c|c|}
\hline Pyrethroid & $\begin{array}{l}\text { Acute oral LD } \\
(\mu \mathrm{g} \text { a.i./bee })\end{array}$ & $\begin{array}{l}\text { Acute contact } \mathrm{LD}_{50} \\
(\mu \mathrm{g} \text { a.i./bee })\end{array}$ \\
\hline Bifenthrin & 0.01 & 0.002 \\
\hline Cypermethrin & 0.03 & 0.02 \\
\hline Deltamethrin & 0.08 & 0.001 \\
\hline Permethrin & 0.03 & 0.1 \\
\hline
\end{tabular}




\subsection{Toxicity to humans}

Short-term symptoms of pyrethroids on humans includes irritation in nasal cavity, facial irritation on dermal exposure, mouth ulcer, sore throat due to ingestion, various allergies, tremors. Very high dose of pyrethroids like d-phenothrin might cause instant death or coma. While symptoms of chronic toxicity of pyrethroids include memory loss, change in immunity system, behavioral problems, thyroid problem etc. There have been cases of certain estrogenic inactivity like decrease in sperm count, change in sperm mobility among factory workers. Carcinogenicity of pyrethroids have yet not been found in any cases but permethrin was found to cause cancer in one of the study on mammals. Children are most venerable to pyrethroids because of its use as household insecticides and their under developed immune system. Neurodevelopment effects on children have been a major concern over the years. Cases of Lower mental development index, behavioral problems, difficulty in learning and attention deficit disorder in children have been reported in recent years due to longterm exposure to high concentration of pyrethroids. There have been reported deaths in certain cases like a 45year-old man died 3 hours after eating beans and cheese prepared using 10\% Cypermethrin instead of oil. $\mathrm{He}$ developed symptoms within few minutes including tenesmus, prolonged vomiting, diarrhea, convulsion followed by death. ${ }^{[10]}$

\subsection{Toxicity to Environment}

Whenever any insecticide is applied in agriculture fields, it interacts with light, metal salts present in soil etc. Pyrethroid undergoes direct as well as indirect photochemical reaction when interacted with light. In indirect photochemical reaction, a molecule of pyrethroid interacts with some other photo-chemically activated molecule. In both cases there is homolytic fission of bonds to form certain intermediates. Ester hydrolysis and photo-induced cis-trans isomerism are two main reaction which are observed in all pyrethroids molecule. Isomerism is very important reaction considering the change in toxicity as well as insecticidal activity due to the reaction.

Figure 2.3: Photochemical reaction ${ }^{[9]}$

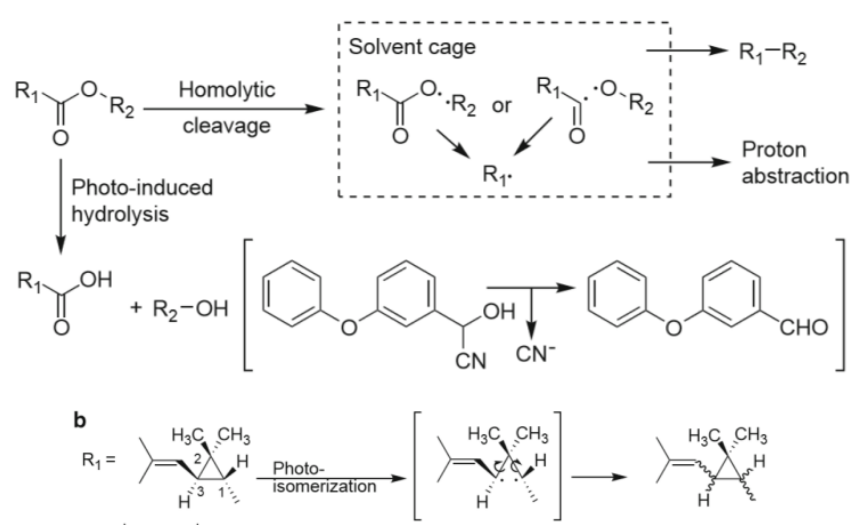

Because of their higher soil absorptivity and moderate metabolic degradation in soil, mobility of pyrethroids in soil is considered to be very limited. Also all pyrethroids are stable under neutral and acidic condition with pH 4-7 but undergoes ester hydrolysis under alkaline condition. Reaction of pyrethroids with air forms oxidation product of the same which might not be active as insecticide.

\subsection{Toxicity of pyrethroid due to synergists}

Synergists are non-toxic compounds which when added to pyrethroids' formulation increases its toxicity for target-insect. Methylenedioxyphenyl type of molecule are most used synergists, for e.g. piperonyl butoxide. They increases the toxicity by reducing the rate of oxidative metabolism of pyrethroids and thus increasing the retention time.

Table 2.4: Effect of synergists on toxicity ${ }^{[8]}$

\begin{tabular}{|c|c|c|}
\hline Treatment & No. of Sensitive mites & No. of Resistant mites \\
\hline Permethrin & 4 & 15 \\
\hline Permethrin + PBO & 4 & 4 \\
\hline Permethrin + DEF & 3.5 & 6 \\
\hline Permethrin + DEM & 2 & 3 \\
\hline
\end{tabular}

Table 2.4 clearly shows that there is decrease in no. of resistant mites due to addition of PBO (piperonyl butoxide), DEM (diethyl maleate), DEF (S,S,S,- tributyl phosphorotrithionate). 


\section{Mechanism of metabolism of pyrethroids}

Oral absorption rates of pyrethroids are generally higher with respect to dermal absorption rates. Although being higher oral absorption rate, alcohol and acid moiety are rapidly and completely excreted into urine and feces within several days. But type II pyrethroids with cyano group shows incomplete excretion and thus higher bio-retention in skin and stomach and thus is more toxic with respect to type I pyrethroids. Major metabolic reactions are ester hydrolysis, oxidation and conjugation in all type of pyrethroids. These reactions occur in two phases, ester hydrolysis and oxidation being phase I reactions while hydrophilic and lipophilic conjugation being phase II reactions. These metabolites of pyrethroids show less acute toxicity than parent compound and thus lead to low mammalian toxicity.

\subsection{Phase I metabolism reaction Ester hydrolysis}

Ester hydrolysis occurs in all type of pyrethroids because of presence of ester linkage which is easy undergo hydrolysis easily giving back acid and alcohol moiety. Ester hydrolysis greatly depends on cis and trans isomerism of alcohol moiety with latter easy to undergo hydrolysis. But the chirality (1S or 1R) at the acid moiety does not affect the rate of hydrolysis. Now, rate of hydrolysis of pyrethroids with primary alcohol (in permethrin) is much faster with respect to that of secondary alcohol like in the case of Cypermethrin.

Figure 3.1: Ester Hydrolysis ${ }^{[3]}$
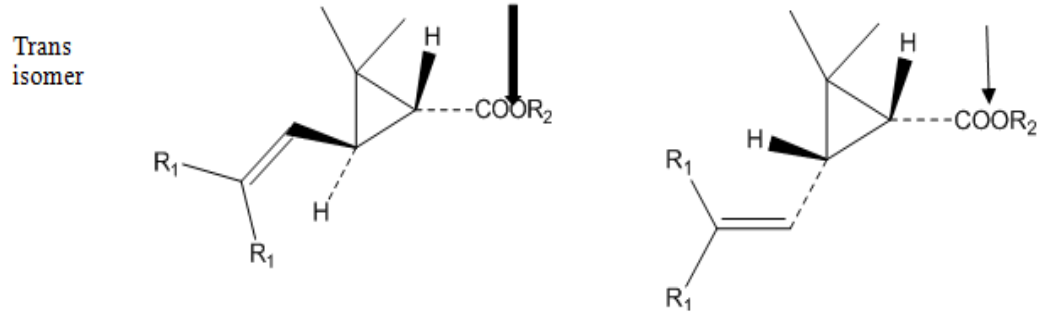

Cis isomer
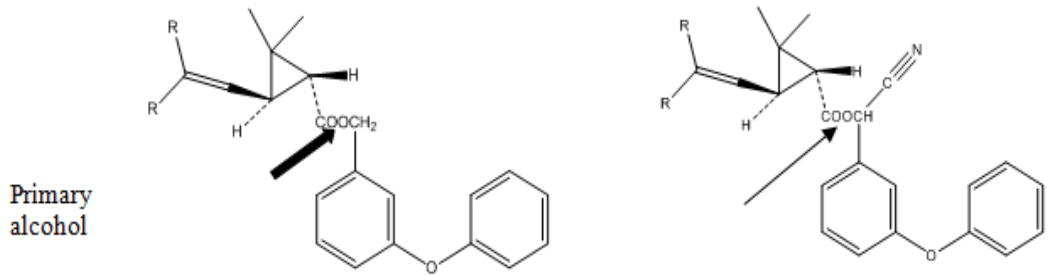

Secondary alcohol

\subsection{Oxidation reaction ${ }^{[7]}$}

Oxidation reaction occurs at several places depending upon the structure of pyrethroids i.e. alcohol and acid moieties. Trans methyl of isobutenyl in chrysanthemic derivative is oxidized preferentially over cis methyl. On alcohol side, 4' position of phenoxy ring undergoes oxidation reaction much faster than other position.

Figure 3.2: Oxidation reaction ${ }^{[3]}$<smiles>C=CC(OC(=O)C1C(C=C(C)C)C1(C)C)c1cccc(Oc2ccc(C)cc2)c1</smiles>

Deltamethrin

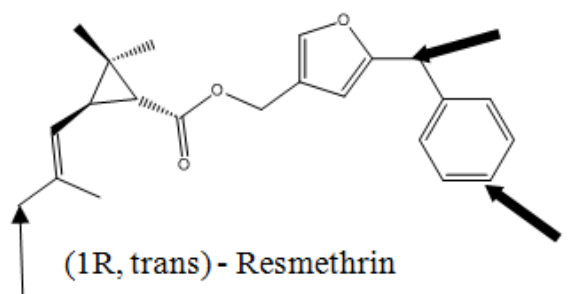




\subsection{Phase II reaction: Hydrophilic Conjugation}

Hydrophilic conjugates found in mammals are glucuronides, sulfates, and amino acid conjugates. 3Phenoxybenzoic acid is one of the most obtained metabolite which can undergo formation of amino acid conjugate. In addition to these, thiocyanate and sulphonic acid conjugates are also obtained in case of Cypermethrin and Tetramethrin respectively. A glycine conjugates are found in sheep, cats and gerbils. Various other conjugates found are taurine conjugate, mercapturic acid conjugate, glycylvaline dipeptide conjugate etc.

Figure 3.3: Hydrophilic conjugates[3]

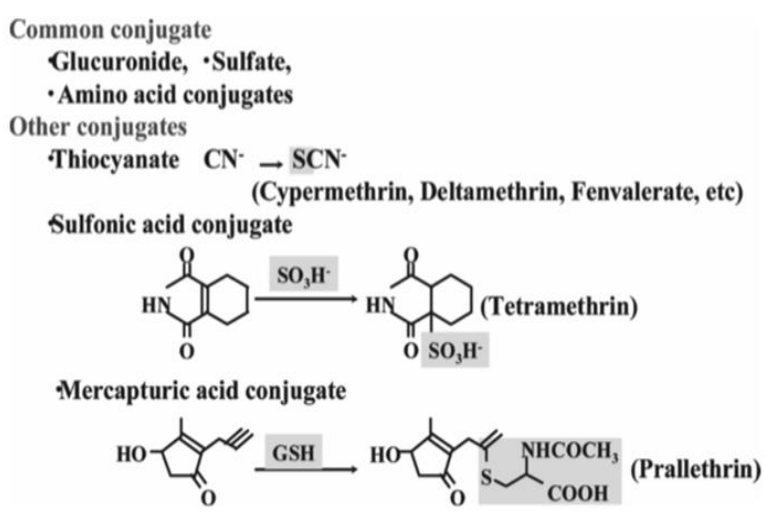

\subsection{Lipophilic conjugation ${ }^{[3]}$}

Various lipophilic conjugates are observed in metabolism mechanism of pyrethroids as shown in figure 3.4. These conjugates tend to remain inside the body for a longer time due to negligible water solubility and thus may affect the organ system of the target insect.

Figure 3.4: Lipophilic conjugates

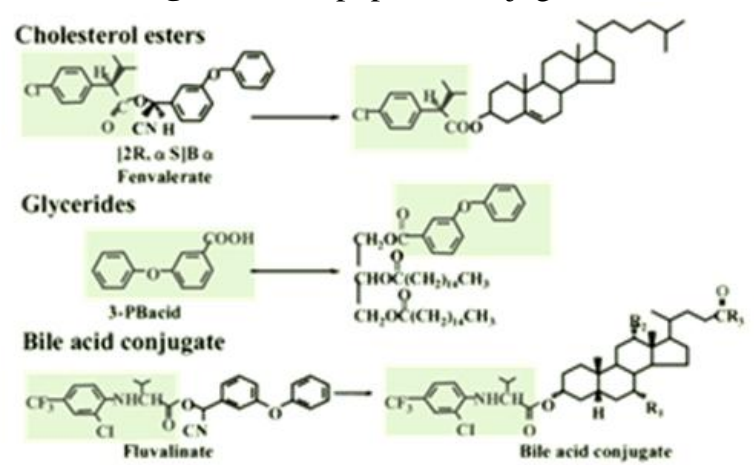

\section{Conclusion}

For more than 40 years, metabolism studies have been carried out extensively, however there have been new pyrethroids molecule introduced into the market showing different metabolites. Also metabolism process rate and their effect in different organs like kidney, liver etc. are yet not known completely. Talking about toxicity of pyrethroids, there should be continuous check to each and every molecule because of increase in resistance to pyrethroids and thus increasing in toxicity of the molecule. Also increasing in laws for environment, there should be continuous efforts to further decrease toxicity of pyrethroids.

\section{References}

[1]. Dr. Bhupinder Khambay, Pyrethroids Insecticides, Pesticide Outlook, 13, 2002 49-54

[2]. F. Antwi, V. P. Reddy, Toxicology effects of pyrethroids on non-target aquatic insects, Environmental Toxicology and Pharmacology, September 2015

[3]. Hideo Kaneko, Pyrethroids: Mammalian Metabolism and Toxicity, J. Agric. Food Chem. 2011, 59, 2786-2791

[4]. The Pesticides Manual, Twelfth edition

[5]. A.J. Thatheyus, A.Deborah Gnana Selvam, Synthetic Pyrethroids: Toxicity and Biodegradation Applied Ecology and Environmental Sciences, 2013, Vol. 1, No. 3, 33-36

[6]. J. J. Schleier \& R. K. Peterson, Green Trends in Insect Control, Ch. 3, Royal Society of Chemistry, 2011, 94-13

[7]. Junshi Miyamoto, Degradation, Metabolism and Toxicity of Synthetic Pyrethroids, EHP, 1976, vol. 14, 15-28 
[8]. C. Pasa, L. Arlian, M. Morgan, R. Gunning, L. Rossiter, D. Holt, S. Walton, S. Beckham, J. McCarthy, Synergists in PyrethroidResistant Scabies Mites, PLOS journal of neglected tropical diseases, January 2009, Volume 3, Issue 1

[9]. Noritada Matsuo \& Tatsuya Mori, Pyrethroids: From Chrysanthemumto Modern Industrial Insecticide, 2012, Ch.1

[10]. Technical Report, Louise Hénault-Ethier, Canadian Association of Physicians for the Environment, 2016 